\title{
MASS MEASUREMENTS IN PROTOPLANETARY DISKS FROM HYDROGEN DEUTERIDE
}

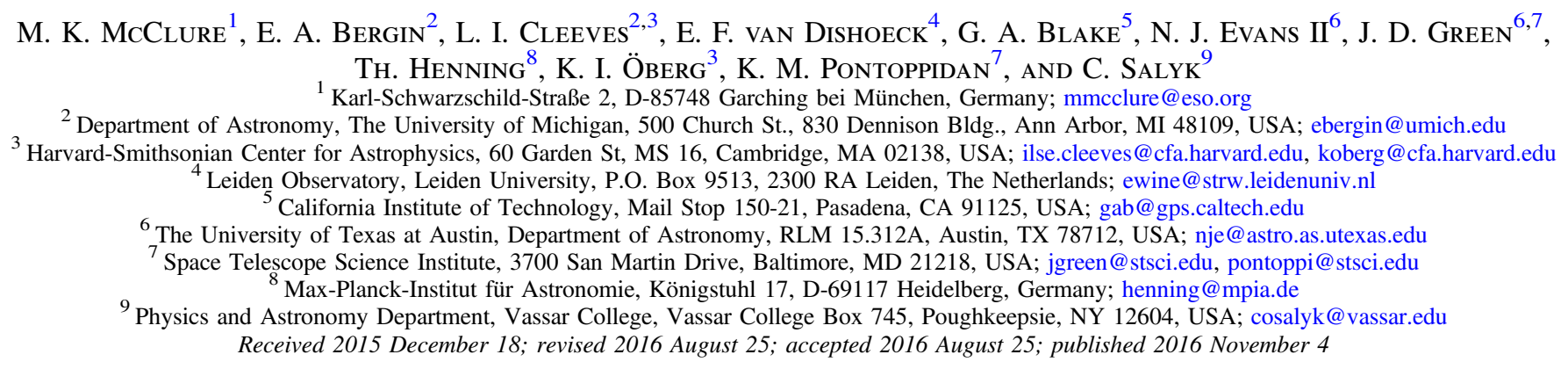

\section{ABSTRACT}

The total gas mass of a protoplanetary disk is a fundamental, but poorly determined, quantity. A new technique has been demonstrated to assess directly the bulk molecular gas reservoir of molecular hydrogen using the HD $J=1-0$ line at $112 \mu \mathrm{m}$. In this work we present a Herschel Space Observatory ${ }^{10}$ survey of six additional T Tauri disks in the HD line. Line emission is detected at $>3 \sigma$ significance in two cases: DM Tau and GM Aur. For the other four disks, we establish upper limits to the line flux. Using detailed disk structure and ray-tracing models, we calculate the temperature structure and dust mass from modeling the observed spectral energy distributions, and we include the effect of UV gas heating to determine the amount of gas required to fit the HD line. The ranges of gas masses are 1.0-4.7 $\times 10^{-2}$ for DM Tau and 2.5-20.4 $\times 10^{-2}$ for GM Aur. These values are larger than those found using $\mathrm{CO}$ for GM Aur, while the CO-derived gas mass for DM Tau is consistent with the lower end of our mass range. This suggests a CO chemical depletion from the gas phase of up to a factor of five for DM Tau and up to two orders of magnitude for GM Aur. We discuss how future analysis can narrow the mass ranges further.

Key words: astrobiology - protoplanetary disks - radiative transfer

\section{INTRODUCTION}

As a fundamental property of protoplanetary disks, the total disk gas mass is of critical importance to our understanding of disk evolution (Armitage 2011). This question, in turn, informs the field of planet formation: the disk gas mass at various ages is taken as an initial condition by planetary population synthesis models (Mordasini et al. 2012). The majority constituent of circumstellar gas, $\mathrm{H}_{2}$, is difficult to observe directly at low temperatures because it lacks a dipole moment and its transitions lie at wavelengths that are difficult to observe. Instead, disk gas masses are typically inferred through one of two proxies: submillimeter observations of either the dusty disk component (Beckwith et al. 1990; Andrews \& Williams 2005, 2007a) or the molecular gas, that is, CO (Dutrey et al. 1996; Williams \& Best 2014).

However, both methods are inherently uncertain. The former assumes specific dust/gas mass ratios, dust opacities, and a disk radius as input, which can be affected by differing grain sizes, compositions, local dust overdensities, or a lack of spatial resolution (Testi et al. 2014, p. 339). The latter assumes a $\mathrm{CO} / \mathrm{H}_{2}$ abundance that is known to vary across the disk due to UV photodissociation in the surface layers and freeze-out of CO in the cold midplane (Dutrey et al. 1997; van Zadelhoff et al. 2001; Reboussin et al. 2015). Moreover, isotope-selective processes need to be taken into account to properly infer disk masses from observations of $\mathrm{C}^{18} \mathrm{O}$ lines (Visser et al. 2009; Miotello et al. 2014), and the models

\footnotetext{
${ }^{10}$ Herschel is an ESA space observatory with science instruments provided by European-led Principal Investigator consortia and with important participation from NASA.
}

assume an overall abundance of carbon in volatile form as an input parameter. All of these uncertainties combined can lead to mass estimates differing by several orders of magnitude (Bruderer et al. 2012; Favre et al. 2013; Bergin et al. 2014; Kama et al. 2016b). A third method has been demonstrated by Bergin et al. (2013) to assess directly the bulk molecular gas reservoir of molecular hydrogen using an isotopologue of $\mathrm{H}_{2}$, hydrogen deuteride (HD); compared with the previously discussed proxies, HD should have a constant abundance relative to $\mathrm{H}_{2}$ throughout the disk. Taking advantage of the wavelength coverage and sensitivity of the Herschel Space Observatory, these authors were able to observe the 1-0 transition of HD at $112 \mu \mathrm{m}$ in the nearest protoplanetary disk, TW Hya. Based on the line strength and detailed chemical modeling, they suggested a lower limit to the disk gas mass for TW Hya of $0.06 M_{\odot}$ or six times the "minimum mass solar nebula" $\left(\mathrm{MMSN}=0.01 M_{\odot}\right.$, Hayashi 1981). That work, however, only encompassed the first object of a larger sample observed with Herschel.

We present the analysis of the remaining sample of six $\mathrm{T}$ Tauri stars, the selection of which is described in Section 2. Through an approach combining disk structure, UV radiation, and gas heating/cooling models, we determine a set of dust and gas density and temperature structures that fit the spectral energy distribution (SED) and HD line emission (Section 3). We find a range of disk masses that fit the observations for the two sources with HD detections (DM Tau and GM Aur, Section 4). In Section 5, we compare our results to CO mass measurements and discuss how the uncertainties in mass might be reduced further. 
Table 1

Observations and Stellar Parameters

\begin{tabular}{|c|c|c|c|c|c|c|}
\hline Parameter & DM Tau & GM Aur & VZ Cha & AA Tau & FZ Tau & LkCa 15 \\
\hline OBSID & 1342239747 & 1342243524 & 1342232613 & 1342239749 & 1342239750 & 1342240148 \\
\hline Date & 2012 Feb 26 & 2012 Mar 25 & 2011 Nov 22 & 2012 Feb 27 & 2012 Feb 27 & 2012 Feb 17 \\
\hline R.A. $(\mathrm{J} 2000)^{\mathrm{a}}$ & 043348.718 & 045510.983 & 110923.790 & 043455.424 & 043231.764 & 043917.796 \\
\hline Decl. $(\mathrm{J} 2000)^{\mathrm{a}}$ & +181009.99 & +302159.54 & -762320.76 & +242853.16 & +242003.00 & +222103.48 \\
\hline$d(\mathrm{pc})$ & 140 & 140 & 160 & 140 & 140 & 140 \\
\hline$T_{\text {eff }}(\mathrm{K})$ & 3720 & 4350 & 3780 & 4060 & 3850 & 4730 \\
\hline$A_{V}(\mathrm{mag})$ & 0.5 & 0.8 & 1.9 & 1.3 & 6.6 & 1.7 \\
\hline$M_{*}\left(M_{\odot}\right)$ & 0.65 & 1.1 & 0.85 & 0.8 & 0.6 & 1.3 \\
\hline$R_{*}\left(R_{\odot}\right)$ & 1.2 & 1.7 & 1.6 & 1.8 & 2.3 & 1.6 \\
\hline$\dot{M}\left(M_{\odot} \mathrm{yr}^{-1}\right)$ & $2 \times 10^{-9}$ & $4.7 \times 10^{-9}$ & $6.2 \times 10^{-8}$ & $6 \times 10^{-9}$ & $3.4 \times 10^{-7}$ & $3.3 \times 10^{-9}$ \\
\hline$i\left(^{\circ}\right)$ & 35 & 55 & $60^{\mathrm{b}}$ & 71 & 75 & 42 \\
\hline$R_{\text {out }}(\mathrm{au})$ & 160 & 300 & $140^{\mathrm{c}}$ & 140 & $140^{\mathrm{c}}$ & 300 \\
\hline
\end{tabular}

Notes.

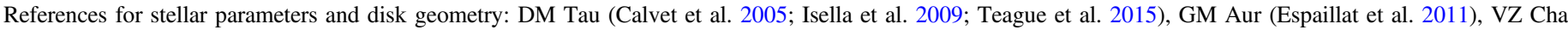
(Manara et al. 2016), AA Tau (Cox et al. 2013; McClure et al. 2015), FZ Tau (Ricci et al. 2010), LkCa15 (Espaillat et al. 2010).

${ }^{\mathrm{a}}$ R.A. and decl. are given in (h $\mathrm{m} \mathrm{s}$ ) and (deg arcmin $\operatorname{arcsec}$ ), respectively.

${ }^{b}$ VZ Cha does not have a measured inclination, so we assume an average value.

${ }^{\mathrm{c}} \mathrm{VZ}$ Cha and FZ Tau do not have measured $R_{\text {out }}$, so we assume a value of $140 \mathrm{au}$.

\section{OBSERVATIONS AND DATA REDUCTION}

For these pioneering observations of this line, there was strong concern about its detectability because the dust continuum of many disks is strong and optically thick at $112 \mu \mathrm{m}$, and the predicted line/continuum ratio was low. Given this issue, the sample was selected from objects that had previous molecular line detections, and all of our targets were selected to have weak continuum emission (below $5 \mathrm{Jy}$ ) at $100 \mu \mathrm{m}$. As an additional factor, our source selection is weighted toward highly settled systems where the dust photosphere is likely below the main layers of gas emission or systems where there is evidence for substantial grain growth (e.g., transition disks); both would lower the $100 \mu \mathrm{m}$ dust optical depth. This can be tracked by the $n_{13-31}$ index, a spectral slope measured between 13 and $31 \mu \mathrm{m}$ by the Spitzer InfraRed Spectrograph (Furlan et al. 2006). However, since the goal of this program was to detect a line, we chose deeper integrations on sources selected to maximize detection rather than conducting a shallow survey of a sample that tests a range of parameter space.

The sample was observed by Herschel (Pilbratt et al. 2010) with the Photodetector Array Camera and Spectrometer (PACS; Poglitsch et al. 2010) through program OT1_ebergin_4 (PI: Bergin) in range spectroscopy mode for $8320 \mathrm{~s}$ per target. The observation IDs are given in Table 1 . PACS is a $5 \times 5$ array of 9 !" $4 \times 9$ !. 4 spatial pixels or "spaxels" at spectral resolution $R \sim 1500-3000$. The nominal pointing rms of the telescope is $2^{\prime \prime}$. The data were reduced using the HIPE interactive pipeline version $13 /$ CalTree 65 , provided by the Herschel Science Center, the most current version at the time of reprocessing during summer 2014. The line-scan mode has toonarrow wavelength coverage (less than $2 \mu \mathrm{m}$ width) to utilize the pipeline "jitter" correction, but other corrections are included. A complete description of the pipeline, along with estimates of precision and efficiency, can be found in Green et al. (2016). The spectra are rebinned by a factor of two before postprocessing.

In Figure 1 we display the reduced spectra of each target, along with a first-order polynomial continuum fit to regions on either side of the HD line. After subtracting the continuum, we measured the emission through fits to a Gaussian line profile
(Figure 2). The full width at half maximum (FWHM) is fixed at $0.115 \mu \mathrm{m}$, according to the instrument profile, while the central wavelength, $\lambda_{c}$, is initially set at $112.072 \mu \mathrm{m}$ (CDMS line list) but allowed to vary within $0.01 \mu \mathrm{m}$ to account for uncertainty in the wavelength calibration. We obtain a best fit to the peak value at the central wavelength position, $F_{0}$, and measure the uncertainty in the value by the rms value of the fit residuals over the line profile. The integrated flux, $F_{\mathrm{int}}$, is taken over the six-channel width of the best-fitting profile, and its uncertainty is calculated from the FWHM, $F_{0}$, and the rms uncertainty. Two of the six targets, DM Tau and GM Aur, show weak but statistically significant HD emission. The peak and integrated fluxes are reported in Table 2. For the four disks in which HD was not detected, the listed integrated fluxes are $3 \sigma$ upper limits.

\section{ANALYSIS}

The amount of emission in the HD line depends on the fractional population of the HD $J=1$ level, which is a function of both the gas temperature and the total disk gas mass. To determine the best-fitting disk mass while taking into account simultaneously the effects of gas temperature, we use three modeling prescriptions to fit the combination of the observed SED (dominated by details of dust structure) and the HD gas line. First, we fit the SED with the D'Alessio et al. (2006) disk structure models to determine the dust density and temperature at each position $\left(\rho_{\text {dust }}, T_{\text {dust }}, M_{\text {dust }}\right)$ and produce a grid of initial gas density distributions and masses $\left(\rho_{\text {gas }}, M_{\text {gas }}\right)$ to be used in determining the gas temperature and disk gas masses (Section 3.1). The densities and temperature structure are used as input to the Bethell \& Bergin (2009) continuum radiative transfer code to calculate the UV flux at each point in the disk (Section 3.2). This information is used with the prescription of Bruderer (2013) to calculate a separate gas temperature structure, $T_{\text {gas }}$. The separate density and temperature structures for the dust and gas are then given as input to RADLite (Pontoppidan et al. 2009), with an assumed abundance of $x_{\mathrm{HD}}=3 \times 10^{-5}$ relative to $\mathrm{H}_{2}$ (Section 3.3). The line emission produced by RADLite is compared with the observed data, and, for the case of the disks with detections, we iterate this 

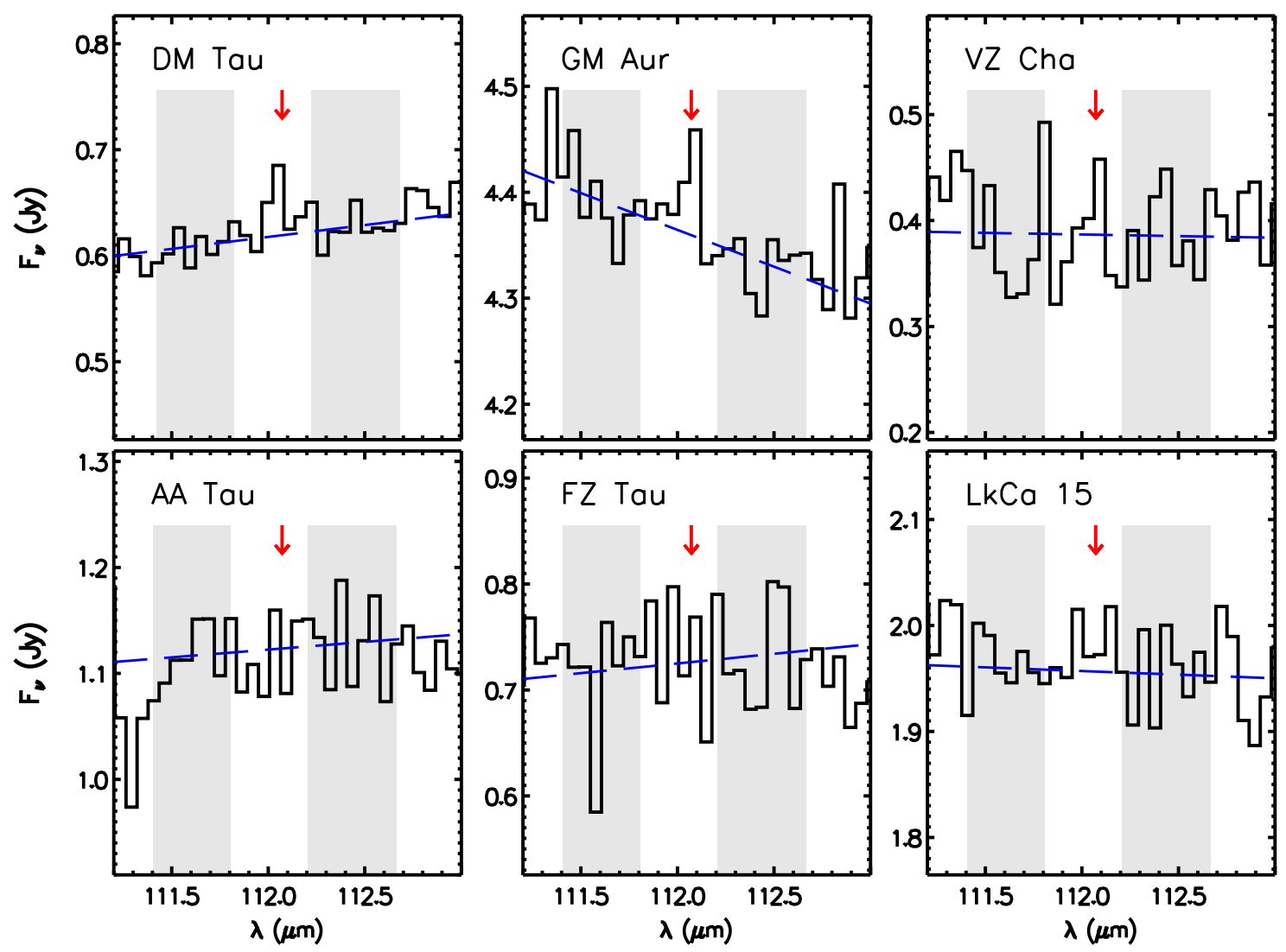

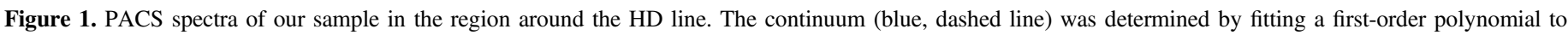
regions on either side of the feature (gray fill). The red arrow indicates the location of the HD line.

procedure over a range of disk temperature structures and gas masses until the line emission fits. We give more detailed descriptions of input to each code below.

\subsection{Initial Disk Structure Model}

To construct the initial disk temperature and density structures, we used the updated D'Alessio Irradiated Accretion Disk (DIAD) 1+1D disk structure code (D'Alessio et al. 2004, 2006), which calculates the structure equations for a gas- and dust-rich disk assuming hydrostatic equilibrium. Heating is provided by both viscous accretion and irradiation by the star and its accretion shock, and it is assumed that the dust and gas temperatures are coupled throughout the disk. This assumption is reasonable for the cooler molecular layers we expect to probe with HD, but ultimately we relax this requirement at a later stage to test this assumption (see Section 3.2). The dust in the disk is distributed between two populations: one in the disk midplane (below $0.1 H$, where $H$ is the gas pressure scale height of the disk) and the other in the disk upper layers (above $0.1 H$ ). The distribution of dust between these layers sets the temperature structure, while the total gas mass is a function of the surface density, disk size, and dust/gas mass ratio. Since we use a physical model for the disk surface density that enforces hydrostatic equilibrium selfconsistently, the dust/gas mass ratio is an input rather than the disk gas mass, and it is not possible to vary the disk temperature and gas mass entirely independently of each other. The input parameters that affect the temperature structure and mass are detailed below.

\subsubsection{Dust/Gas Mass Ratio and Dust Temperature Structure}

The disk temperature structure is strongly affected by the settling of dust grains from the disk upper layers to the midplane, as a reduction in small grains in the upper layers allows stellar radiation to penetrate deeper into the disk. This effect is particularly pronounced for the separate UV gas heating module (Section 3.2), as discussed in Section 4.1. The amount of dust depletion is measured relative to the "standard" dust/gas mass ratio: $\epsilon_{\text {small }}=\chi_{\text {upperlayers }} / \chi_{\text {standard }}$, where $\chi_{\text {standard }}$ is determined from the dust species mass fractions. Larger values of $\epsilon_{\text {small }}$ indicate less dust depletion; for example, $\epsilon_{\text {small }}=0.5$ indicates $50 \%$ of the dust is depleted, while a value of 0.01 indicates that $99 \%$ is depleted. The dust/gas ratio in the midplane is enhanced by the solids that settle out of the upper layers. The value of dust enhancement, described by $\epsilon_{\text {big }}=\chi_{\text {midplane }} / \chi_{\text {standard }}$, is coupled to $\epsilon_{\text {small }}$ so as to conserve mass vertically at a given radius (see Table 3 of D'Alessio et al. 2006).

The dust in our model has a power-law size distribution, from a minimum size of $0.005 \mu \mathrm{m}$ to a maximum size $a_{\max }$ with a power of -3.5 (Mathis et al. 1977). In the midplane, $a_{\max }$ is fixed at $1 \mathrm{~mm}$. In the upper layers, it is fixed at the values found by Espaillat et al. (2011) for DM Tau, GM Aur, and $\mathrm{LkCa} 15$ and allowed to vary for the other disks. The dust grain species and opacities differ from D'Alessio et al. (2006). For five of the six disks, we use only silicates and graphite. The silicate opacities are taken to be those that best fit the Spitzer IRS spectra in Sargent et al. (2009): amorphous, glassy olivine and pyroxene (Jaeger et al. 1994; Dorschner et al. 1995) or crystalline forsterite and enstatite (Chihara et al. 2002; Sogawa 


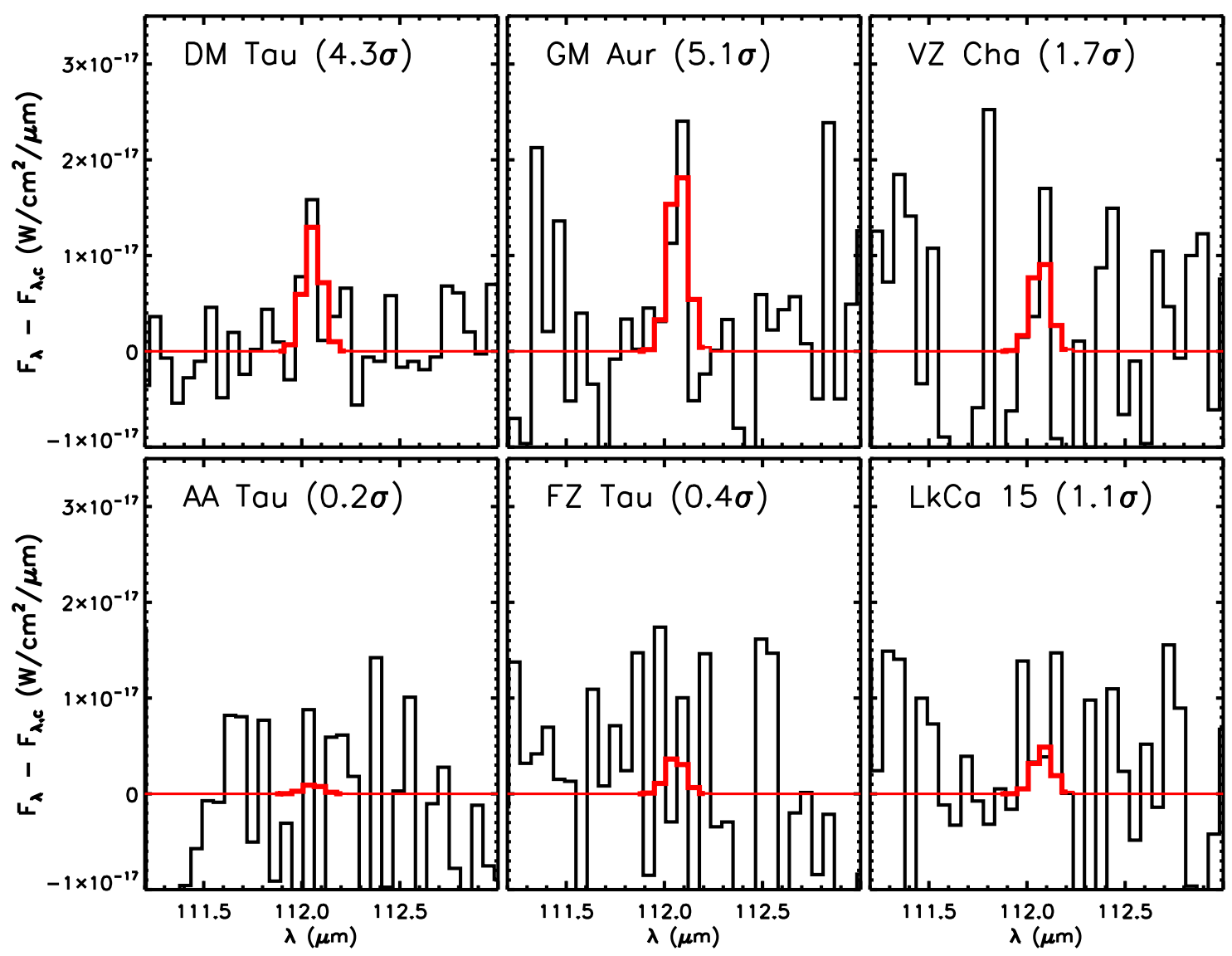

Figure 2. Gaussian line fits (red) to the continuum-subtracted spectra (black). The FWHM and central wavelength were fixed at the instrumental and theoretical values, respectively. The thicker red line indicates the region over which the rms deviation of the residuals is measured. The $1 \sigma$ uncertainty calculation is described in Section 2 .

Table 2

Measured Line Fluxes

\begin{tabular}{lccc}
\hline \hline Star & $F_{0}$ & $F_{\text {int }}$ & $\mathrm{S} / \mathrm{N}$ \\
& $\left(\times 10^{-17} \mathrm{~W} \mathrm{~m}^{-2} \mu \mathrm{m}^{-1}\right)$ & $\left(\times 10^{-18} \mathrm{~W} \mathrm{~m}^{-2}\right)$ & \\
\hline DM Tau & $1.30 \pm 0.43$ & $1.6 \pm 0.4$ & 4.3 \\
GM Aur & $2.00 \pm 0.55$ & $2.5 \pm 0.5$ & 5.1 \\
VZ Cha & $1.00 \pm 0.81$ & $<2.1^{\mathrm{a}}$ & $\cdots$ \\
AA Tau & $0.10 \pm 0.85$ & $<2.2^{\mathrm{a}}$ & $\cdots$ \\
FZ Tau & $0.40 \pm 1.40$ & $<3.6^{\mathrm{a}}$ & $\cdots$ \\
LkCa 15 & $0.50 \pm 0.66$ & $<1.7^{\mathrm{a}}$ & $\cdots$ \\
\hline
\end{tabular}

Notes. Each flux was measured using a Gaussian fit with a fixed FWHM of $0.115 \mu \mathrm{m}$ and central wavelength of $112.07159 \mu \mathrm{m}$. Column 2: peak flux at central wavelength, with residual rms uncertainty; Column 3: integrated flux ${ }^{a} 3 \sigma$ upper limit.

et al. 2006) with a mass fraction of 0.004 relative to the gas. The opacity and mass fraction of 0.0025 for graphite is taken from Draine \& Lee (1984). For these disks, $\chi_{\text {standard }}=0.0065$. Water ice is included only for AA Tau, in which it has been detected through spectral features (Chiang et al. 2001; McClure et al. 2015). In that case, the water ice mass fraction is 0.002 , $\chi_{\text {standard }}=0.0085$, and the composite ice opacities are taken directly from McClure et al. (2015).

\subsubsection{Surface Density}

In addition to changing the temperature structure, varying the surface density, $\Sigma$, also changes $M_{\text {gas }}$, the disk gas mass.
Table 3

Sample UV Properties

\begin{tabular}{lccc}
\hline \hline Star & $\begin{array}{c}L_{\text {acc }} \\
\left(L_{\odot}\right)\end{array}$ & $\begin{array}{c}7 L_{\mathrm{FUV}} \\
\left(L_{\odot}\right)\end{array}$ & Ref. \\
\hline DM Tau & $\ldots$ & $4.02 \times 10^{-3}$ & 1 \\
GM Aur & $\ldots$ & $1.85 \times 10^{-3}$ & 1 \\
VZ Cha & $\ldots$ & $\ldots$ & $\ldots$ \\
AA Tau & $\ldots$ & $1.99 \times 10^{-3}$ & 1 \\
FZ Tau & $4.64 \times 10^{-1}$ & $1.10 \times 10^{-2}$ & 3 \\
LkCa 15 & $2.51 \times 10^{-2}$ & $9.83 \times 10^{-4}$ & 2 \\
\hline
\end{tabular}

Note. References: (1) Yang et al. (2012), Table 4; (2) Yang et al. (2012), Table 2; (3) Ricci et al. (2010), from $\dot{M}=7.2 \times 10^{-8} M_{\odot} \mathrm{yr}^{-1}$. For comparison, Bergin et al. (2013) used $L_{\mathrm{FUV}}=1.86 \times 10^{-3} L_{\odot}$ for TW Hya.

The surface density of the disk is proportional to the mass accretion rate divided by the Shakura \& Sunyaev (1973) $\alpha$ parameter (which describes the disk viscosity): $\Sigma \propto \dot{M} / \alpha$. The dust emission is directly related to the temperature, opacity, and mass of dust, where the dust emission becomes optically thin at millimeter wavelengths: $F_{\nu} \propto T_{\text {dust }} \kappa_{\nu} M_{\text {dust }}$. These physical quantities are related to the model input parameters: $T_{\text {dust }}$ depends on $\epsilon_{\text {small }}, \kappa_{\nu}$ is a function of the assumed dust species and dust/gas mass ratio, and $M_{\text {dust }}$ is determined by integrating $\Sigma_{\text {dust }}$ from the inner to outer disk radii.

By taking constraints from observations on $\dot{M}$ and $R_{\text {out }}$ (as discussed in Section 3.1.3), using the McClure et al. (2013) 
Table 4

SED Model Fits

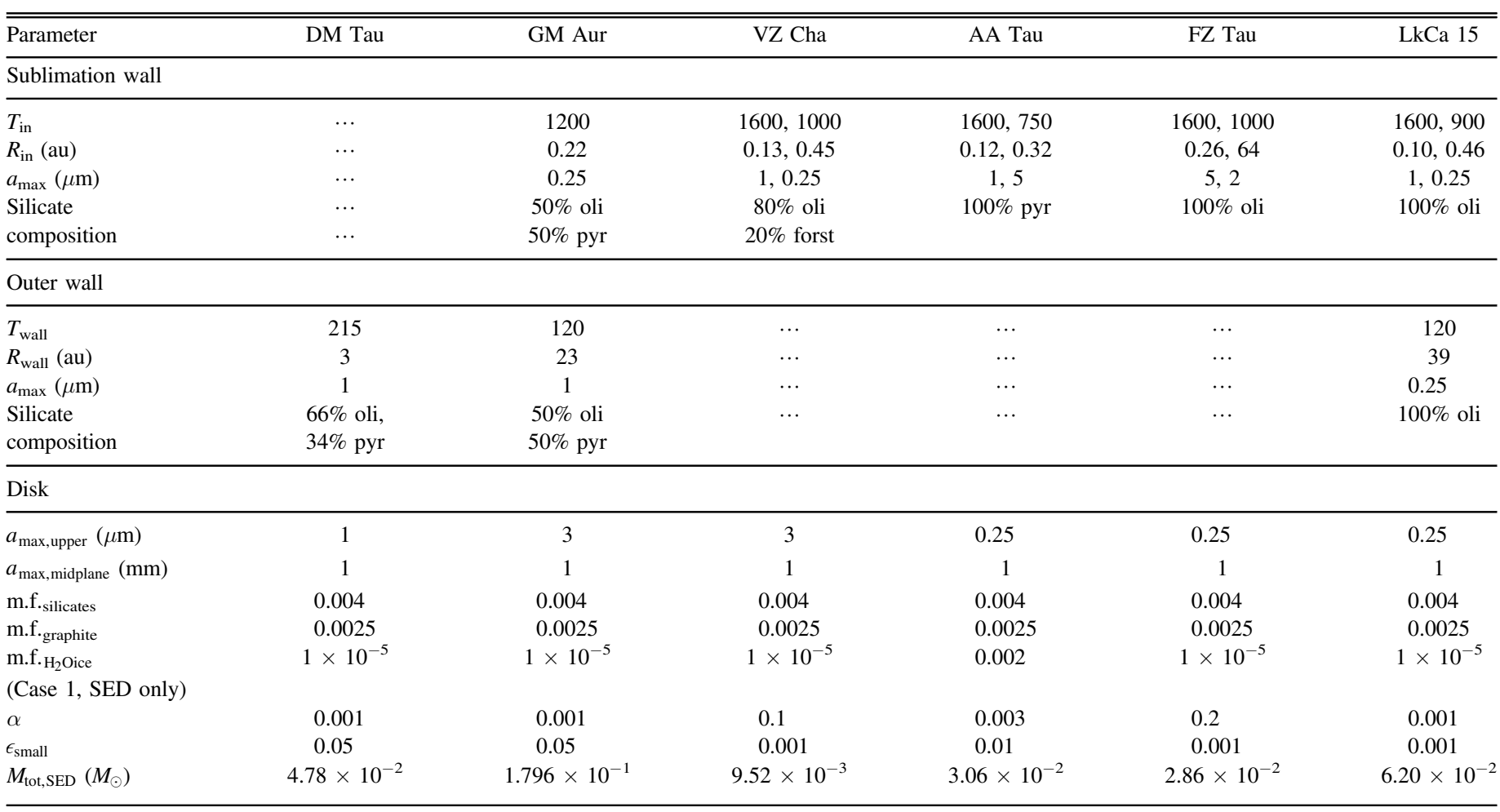

Note. The assumed stellar and accretion properties and disk geometries are given in Table 1. Dust components are (oli)vine, (pyr)oxene, and (forst)erite. The abbreviation "m.f." indicates the mass fraction of a particular grain species relative to the gas. Total masses are given for the dust/gas ratio case where the vertical dust mass was conserved at each radius. The inner silicate sublimation rim is fit with the two-layer model description given by McClure et al. (2013), and we refer the reader to this work for more details. $M_{\text {tot }}$ is defined as the sum of the gas and dust masses.

prescription for a two-layer wall to fit $R_{\text {in }}$, and fixing the dust properties, we can reduce the variable input parameters to the dust/gas ratio in the upper layers $\left(\epsilon_{\text {small }}\right), \alpha$, and the dust/gas ratio at the midplane $\left(\epsilon_{\text {big }}\right)$. These parameters produce a family of models that fit the (sub)millimeter photometry with the same total dust mass, $M_{\text {dust }}$, but with a range of values for the gas mass, $M_{\mathrm{gas}}$.

\subsubsection{Observables}

Certain parameters were fixed to observed values, which are given in Table 1 . The mass accretion rate, $\dot{M}$, is taken to be that of the disk onto the star and is assumed to be constant throughout the disk. We do not account for observed variability about an average value for $\dot{M}$, which can be almost an order of magnitude over several months in the same object (e.g., GM Aur, Ingleby et al. 2015). However, because of the relationship between $\Sigma, \dot{M}$, and $\alpha$, for the same disk structure, $\alpha$ could be varied to compensate for any change in the average $\dot{M}$.

Where observations are available, we take the disk outer radius, $R_{\text {out }}$, to be that of the observed millimeter grains, rather than the larger radius defined by gas observations (Panić et al. 2009; Andrews et al. 2012). It is not clear what effect the removal of large dust grains from the outer disk would have. Over time, removal of the largest grains could lower the concentration of small grains in the disk upper layers, cooling the disk beyond the radius defined by millimeter grains. Since our model does not allow for different gas and dust radii, we truncate the disk structures at the millimeter grain radius to avoid introducing an artificial contribution to the HD emission from the (mostly dust-free, by mass) regions beyond this point. This does not affect our SED fitting, which depends primarily on the dust distribution. For the HD emission, Bergin et al. (2013) found for TW Hya that 90\% of the HD emission originated within $100 \mathrm{au}$, so although there is gas outside the millimeter grain radius, it should not contribute substantially to the line emission.

\subsection{Gas Temperature}

To decouple the gas temperature, $T_{\text {gas }}$, from the dust temperature, $T_{\text {dust }}$, we need to account for heating by UV radiation. To compute the UV radiation field at each point in the disk, we ran the Bethell \& Bergin (2009) code on the bestfitting 1+1D disk structures. We assumed an input stellar UV spectrum of TW Hya $\left(L_{\mathrm{FUV}}=1.86 \times 10^{-3} L_{\odot}\right.$, Bergin et al. 2013), scaled according to the UV luminosity of each target, as given in Table 3. Where it was missing, we calculated the UV luminosity from the mass accretion rate or accretion luminosity, assuming the relationship between $L_{\mathrm{acc}}$ and $L_{\mathrm{FUV}}$ given by Yang et al. (2012). The UV radiation field was combined with the disk gas density to calculate $\Delta T_{\text {gas }}$ according to a prescription described in the appendix of Bruderer (2013), which is calibrated from detailed thermochemical models that are introduced in Bruderer et al. (2012). We compare the differences in results obtained with $T_{\text {gas }}=T_{\text {dust }}$ and $T_{\text {gas }}>T_{\text {dust }}$ in Section 4. 

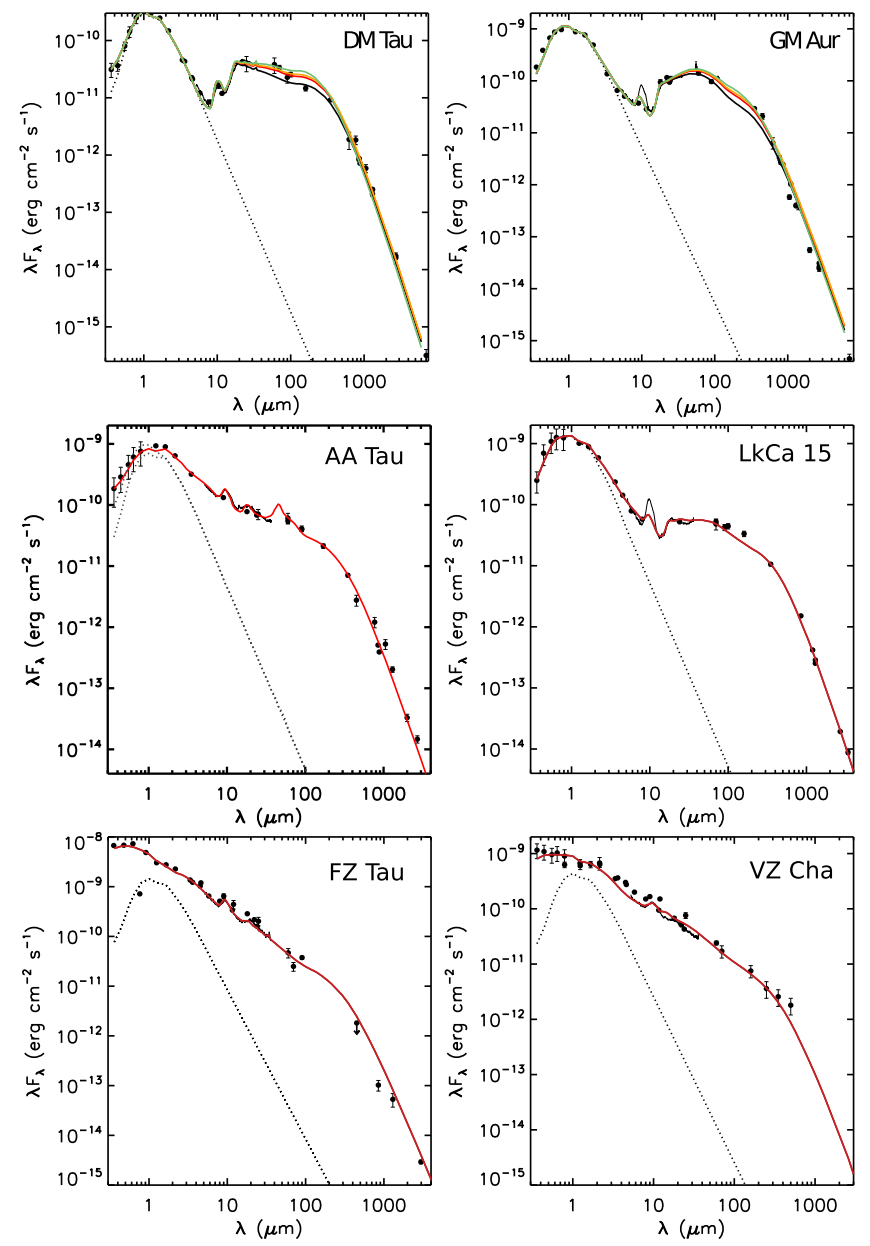

Figure 3. Best-fitting 1+1D models to the spectral energy distributions of our sample. Optimized values for input parameters are given in Table 4. For the two disks with HD detections, DM Tau and GM Aur, we indicate the effect of varying the degree of dust depletion from the disk upper layers, $\epsilon_{\mathrm{small}}$, on the SED fit. For these two disks, models are $\epsilon_{\text {small }}=0.5$ (green), 0.1 (orange), 0.05 (red), and 0.01 (black). References for the photometry are Kenyon \& Hartmann (1995), Herbig \& Bell (1988, p. 3), 2MASS (Cutri et al. 2003), DENIS (Carpenter et al. 2002), WISE (Cutri et al. 2012), AKARI IRC (Ishihara et al. 2010), Spitzer IRAC (Luhman et al. 2008, Spitzer Enhanced Imaging Products), Gräfe et al. (2011), PACS (Winston et al. 2012; Howard et al. 2013), AKARI FIS, Spitzer MIPS (Spitzer Enhanced Imaging Products), IRAS (FSC, PSC Moshir et al. 1990; Helou \& Walker 1988, pp. 1-265, respectively), Adams et al. (1990), Andrews \& Williams (2005, 2007b, 2008), Andrews et al. (2011), Beckwith \& Sargent (1991), Beckwith et al. (1990), Dutrey et al. (1996, 1998), Duvert et al. (2000), Kitamura et al. (2002), Guilloteau et al. (2011), Isella et al. (2009), Osterloh \& Beckwith (1995), Ricci et al. (2010), Rodmann et al. (2006), and Weintraub et al. (1989).

\subsection{Gas Line Emission}

The dual temperature and density structures, $T_{\mathrm{gas}}, \rho_{\mathrm{gas}}, T_{\mathrm{dust}}$, and $\rho_{\text {dust }}$, and the dust opacities were passed into the RADLite line radiative transfer code (Pontoppidan et al. 2009). We took a constant $\mathrm{HD}$ abundance throughout the disk of $x_{\mathrm{HD}}=$ $3 \times 10^{-5}$ relative to $\mathrm{H}_{2}$, assuming $\mathrm{HD} / \mathrm{H}_{2}$ has twice the $\mathrm{D} / \mathrm{H}$ value of $x_{D}=1.5 \times 10^{-5}$ within the nearest $100 \mathrm{pc}$ (Linsky 1998), as suggested by Bergin et al. (2013). As for the case of TW Hya, a lower HD abundance than that assumed here would imply larger final disk masses. Since molecular hydrogen does not freeze out, and there are relatively few ways to preferentially sequester it in other molecules or on grains on disk-wide scales, the constant-abundance assumption is reasonable.
Table 5

Best-fitting Dust and Gas Masses, Cases 1 and 2

\begin{tabular}{lccc}
\hline \hline Value & DM Tau & GM Aur & Reference \\
\hline$M_{\text {dust }}\left(M_{\odot}\right)$ & $2.9 \times 10^{-4}$ & $1.25 \times 10^{-3}$ & $\ldots$ \\
$M_{\text {gas }}\left(M_{\odot}\right)$ & $(1.0-4.7) \times 10^{-2}$ & $(2.5-20.4) \times 10^{-2}$ & $\ldots$ \\
$\epsilon_{\text {small }}$ & $0.02-0.04$ & $0.06-0.1$ & $\ldots$ \\
$\alpha$ & $0.001-0.005$ & $0.001-0.005$ & $\ldots$ \\
$\epsilon_{\text {big }}$ & $12.4-50$ & $11.4-75$ & $\ldots$ \\
\hline$M_{\text {dust,lit. }}\left(M_{\odot}\right)$ & $1.5 \times 10^{-4}$ & & 2 \\
$M_{\text {gas, lit. }}\left(M_{\odot}\right)$ & $1.4 \times 10^{-3}$ & $\leqslant 0.35 \times 10^{-3}$ & 1 \\
& $9.0 \times 10^{-3}$ & & 2 \\
\hline
\end{tabular}

Notes. The dust/gas mass ratio for each region (disk upper layers and midplane) is $\epsilon \times 0.0065$.

References. (1) Dutrey et al. (1996), (2) Williams \& Best (2014).

RADLite then calculates how the HD energy levels are populated relative to the total amount of HD at each point, assuming LTE excitation. During the ray tracing, RADLite also takes into account the dust optical depth when calculating the emergent line emission. The output spectra were resampled to the PACS instrument resolution $\left(300 \mathrm{~km} \mathrm{~s}^{-1}\right.$ at $\left.112 \mu \mathrm{m}\right)$ and integrated over the emission line to be compared with the data. For the two disks with HD detections, we compared the line fluxes calculated for several temperature structures and values of $M_{\mathrm{gas}}$ with the observed line fluxes to determine the bestfitting gas masses.

\section{RESULTS}

To initiate the modeling sequence, we take the following approach. First we fit the dust SEDs of all six disks using models for which mass is conserved in a vertical column, which we call Case 1 . These models allow us to determine the disk dust mass and an acceptable range of temperature structures. We explored the following parameter space: $1 \geqslant \epsilon_{\text {small }} \geqslant 0.001,0.1 \geqslant \alpha \geqslant 0.0001$. Since mass is conserved vertically, $\epsilon_{\text {big }}$ is tied directly to $\epsilon_{\text {small }}$ and does not freely vary. The fit to the submillimeter photometry produces a bestfitting value for $\alpha$, while the fit to the mid- and far-infrared region determines the dust depletion in the disk upper layers, $\epsilon_{\text {small }}$, and the maximum dust grain size in the disk upper layers is constrained by the mid-infrared spectral features. For the four disks with nondetections, we continue the analysis with a single Case 1 model with a value for $\epsilon_{\text {small }}$ that best fits the SED, while for DM Tau and GM Aur we take an additional family of Case 1 models with a range of $\epsilon_{\text {small }}$ about the best-fitting value to test the impact on the HD line emission of varying the temperature structure. All Case 1 models for the six disks are carried forward through the gas temperature and HD line flux calculations in Sections 3.2 and 3.3.

For the best-fitting range of values of $\epsilon_{\text {small }}$ for DM Tau and GM Aur, we then vary the disk surface density via the $\alpha$ parameter to explicitly test the acceptable range of gas masses, which we call Case 2. This required fixing the disk dust mass, to maintain the fit to the (sub)millimeter photometry, by enhancing the dust/gas ratio at the disk midplane via the $\epsilon_{\mathrm{big}}$ parameter. Doing so relaxes the mass conservation requirement assumed in Case 1, as $\epsilon_{\text {big }}$ is allowed to vary independently from $\epsilon_{\text {small }}$, with values between 12.5 and 100. Each decrease in the surface density corresponds to an increase in $\alpha$ and an 


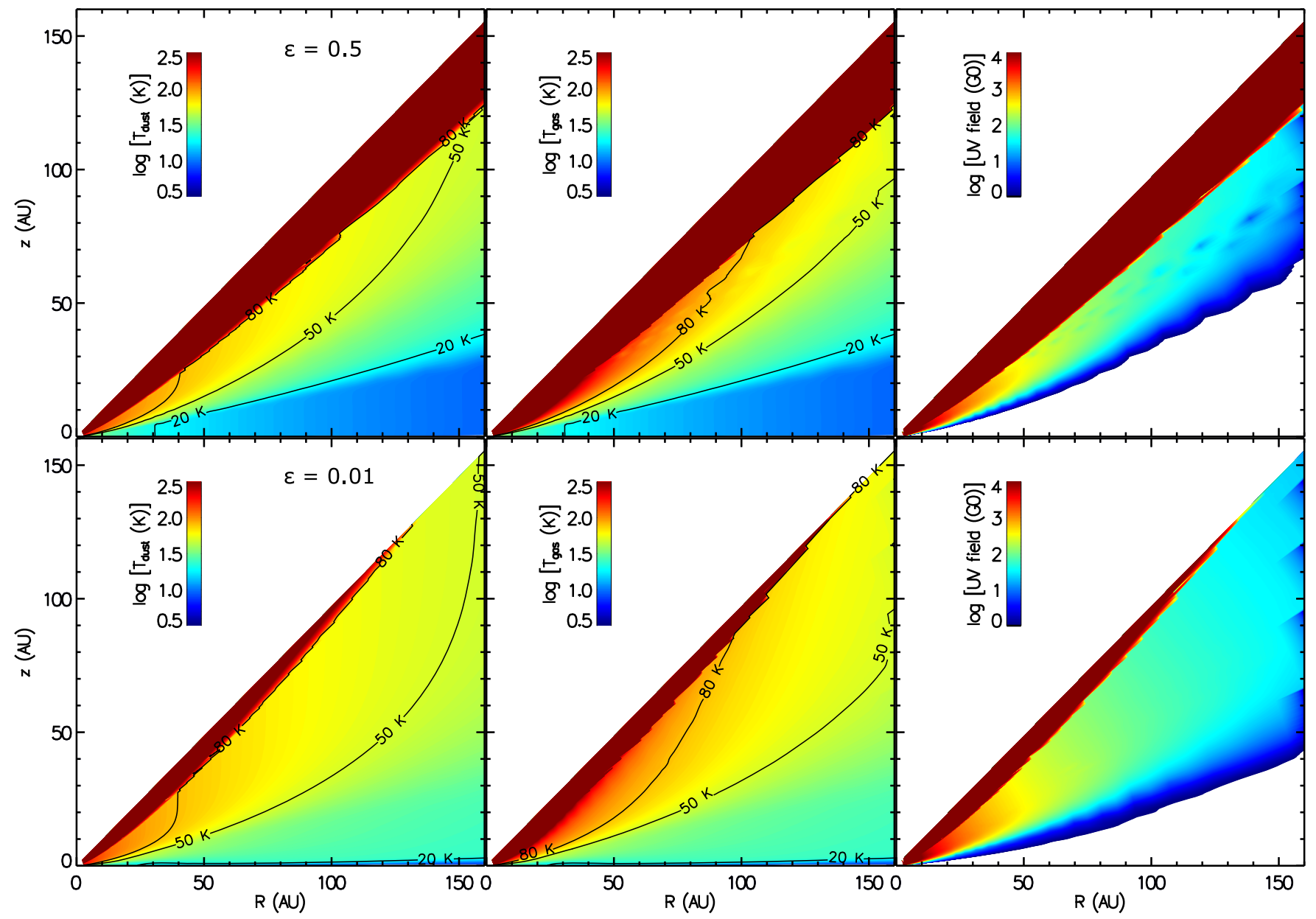

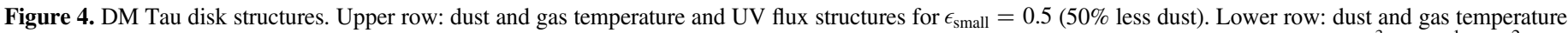

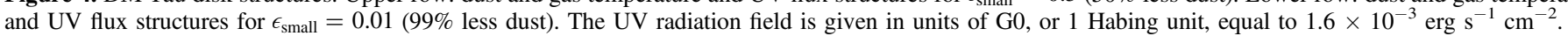

increase in the midplane dust concentration to maintain the fit to the submillimeter photometry. A local enhancement of dust at the midplane is not unphysical because dynamical processes such as radial drift or dust filtration at the edges of gaps (Zhu et al. 2012; Testi et al. 2014, p. 339) could enhance the dust concentration in the midplane interior to the outer radius defined by the millimeter-sized grains. Then for each model in the Case 1 temperature grid, there is a family of Case 2 models with decreasing gas mass. We carry forward all of the Case 2 models through the gas temperature and HD line flux calculations. Combined with the Case 1 models for these disks, we can then use the HD line emission to select the bestfitting range of gas masses.

Below we discuss how varying the dust depletion, gas heating, and optical depth affects the disk structures, line emission, and disk gas masses, using DM Tau and GM Aur as examples. The best-fitting values found for these parameters are given in Table 4 for all six disks, and the SED fits for the two disks with detections are shown in Figure 3. The SEDs of the nondetections are given in Figure 3 and discussed in Section 4.4.

\subsection{Case 1: Impact of Disk Temperature Structure on HD Line Emission}

The effect on the dust temperature structure of varying the dust depletion factor is significant. A smaller dust opacity in the upper layers causes the stellar radiation to be deposited at lower disk altitudes, cooling the uppermost disk layer and warming the lower disk layers (although not the midplane itself). In the left-most panels of Figures 4 and 5, by volume $30 \%$ of the disk is colder than $20 \mathrm{~K}$ in the less-depleted, $\epsilon_{\text {small }}=0.5$ model compared with $<2 \%$ of the more-depleted, $\epsilon_{\text {small }}=0.01$ model. The increased dust depletion from the upper layers manifests in the SEDs as a lower overall flux and a bluer slope near $100 \mu \mathrm{m}$, as seen in Figure 3. In this figure, a range in dust depletion from $50 \%$ to $99 \%$ relative to the standard dust/gas ratio fits similarly well within the error bars, with a formal best-fitting value of $\epsilon_{\text {small }}=0.05_{-0.04}^{+0.05}$, or a $95 \%$ depletion, for both GM Aur and DM Tau. The uncertainty comes from the fact that DM Tau and GM Aur are transitional (i.e., their millimeter emission displays evidence of an inner clearing close to the star), and the frontally illuminated inner edge of the dusty disk dominates the mid-infrared emission and into the far-infrared regime. Consequently, the impact on their SEDs from varying the degree of dust depletion is less pronounced than for full disks. We are ultimately able to put firmer constraints on the value for $\epsilon_{\text {small }}$ through the comparison with the HD line flux below.

Heating by UV radiation also changes the gas temperature structure substantially, as seen in the upper left and center panels of Figures 4 and 5, which show the dust and gas temperatures, respectively. However, the increase in temperature occurs mainly in the disk upper layers above the $50 \mathrm{~K}$ 


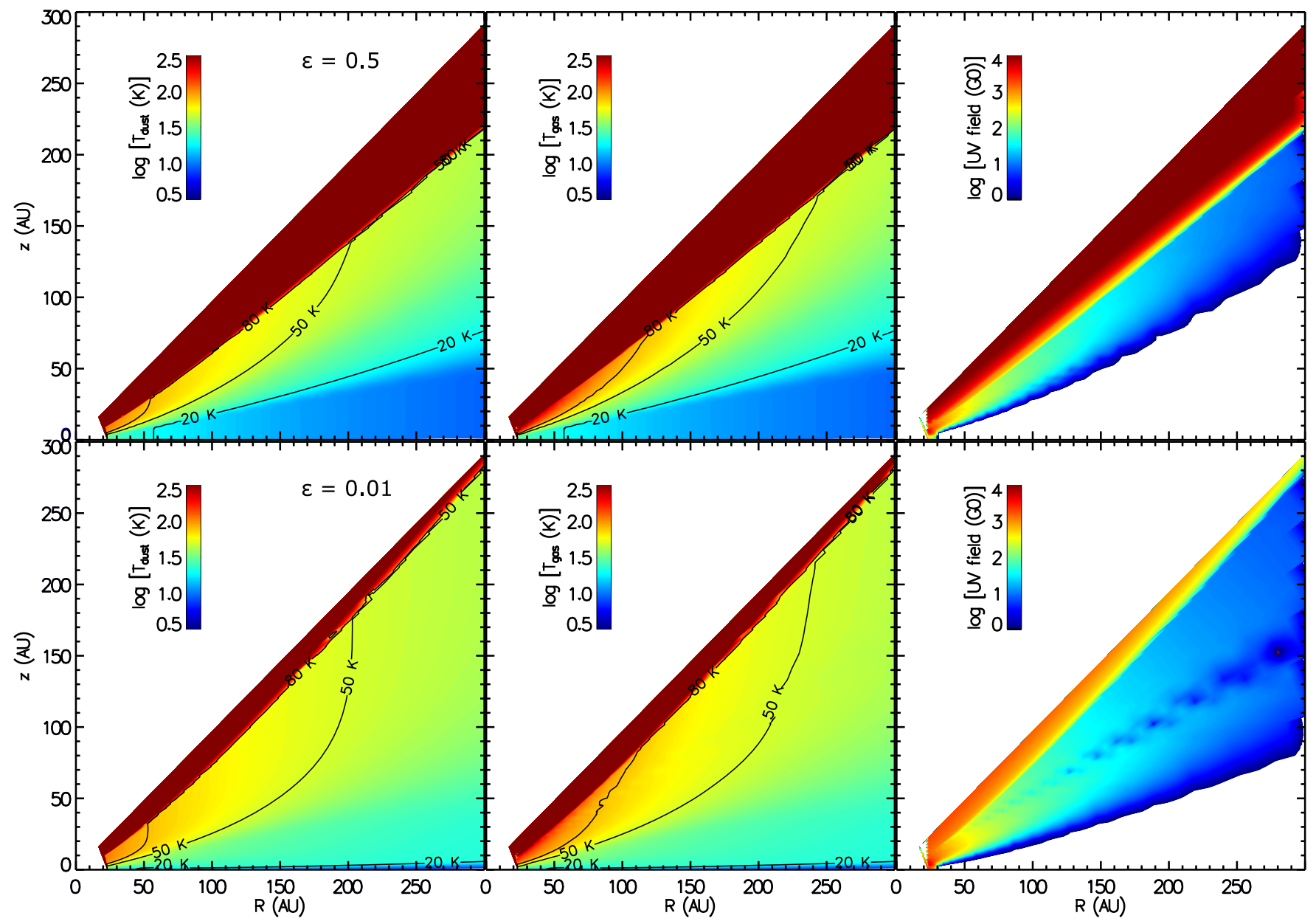

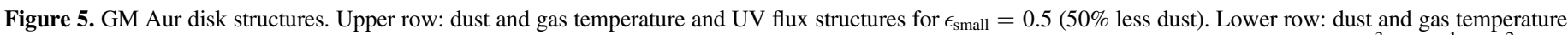
and UV flux structures for $\epsilon_{\text {small }}=0.01$ (99\% less dust). The UV radiation field is given in units of G0, or $1 \mathrm{Habing}^{\mathrm{n}} \mathrm{unit}$, equal to $1.6 \times 10^{-3}$ erg $\mathrm{s}^{-1} \mathrm{~cm}^{-2}$.

isotherm. This is because the UV radiation field is attenuated to interstellar values just below the $50 \mathrm{~K}$ isotherm, as seen in the upper right panel of Figures 4 and 5, so the gas is not heated efficiently at lower disk altitudes. As discussed in Bergin et al. (2013), the HD emission line strength depends both on the temperature of that gas and the total mass in HD, as warmer gas populates the $J=1$ upper state more relative to the $J=0$ level, compared with cooler gas. The fractional populations are compared in the left column of Appendix Figures 7 and 8, in addition to the total gas density and density of HD in the $J=1$ level. For the model with less dust depleted, the $J=1$ density is clearly bimodal, with a strong contribution in the midplane, where the total gas density is strongest, and a spur continuing into the disk upper layers, where the fractional population is highest. This pattern is similar to that found for TW Hya by Bergin et al. (2013). In the more depleted model, however, the $J=1$ level is populated closer to the midplane, so the $J=1$ density is more reflective of the entire disk density structure than it is in the less depleted model.

As a result, the line emission is an order of magnitude stronger in the most depleted model compared with the least depleted. In Figure 6, we compare the integrated line flux as a function of gas mass for Case 1 with $T_{\text {gas }}>T_{\text {dust }}$ and $T_{\text {gas }}=T_{\text {dust }}$. The higher gas temperature produces only a 4 to $6 \%$ increase in the line flux, so we do not consider further the gas temperature separately. The HD line flux provides further constraints on both the value of $\epsilon_{\text {small }}$ and the gas mass. Within the observational uncertainties, the new best-fitting value of the dust depletion parameter $\epsilon_{\text {small }}$ is $0.03 \pm 0.01$ and $0.08 \pm 0.02$ for DM Tau and GM Aur, respectively. The new best-fitting value corresponds to a decrease in mass for DM Tau and an increase in mass for GM Aur. However, the difference in line flux between the Case 1 models is due mainly to the distribution of dust and the temperature structure rather than the mass.

\subsection{Case 2: Effect of Varying the Surface Density on HD Line Emission}

To test directly the impact of gas mass on the line flux, we varied the surface density by increasing $\alpha$ and enhancing the dust/gas mass ratio at the midplane as described above and compared the output line fluxes with the data and Case 1 models in Figure 6. Changing the dust/gas ratio at the midplane produces some variation in the temperature structure, but it is small compared with the effect of changing the dust/ gas ratio in the upper layers of the disk.

The HD line flux varies directly with mass, as we expected. For DM Tau and GM Aur, the range of masses consistent with the observed line is broad: half an order of magnitude and a full order of magnitude, respectively, with $d / g$ at the midplane at $\sim 0.5-0.07$. However, the line flux is more sensitive to changes in gas mass when the upper layers are more depleted in dust, as 

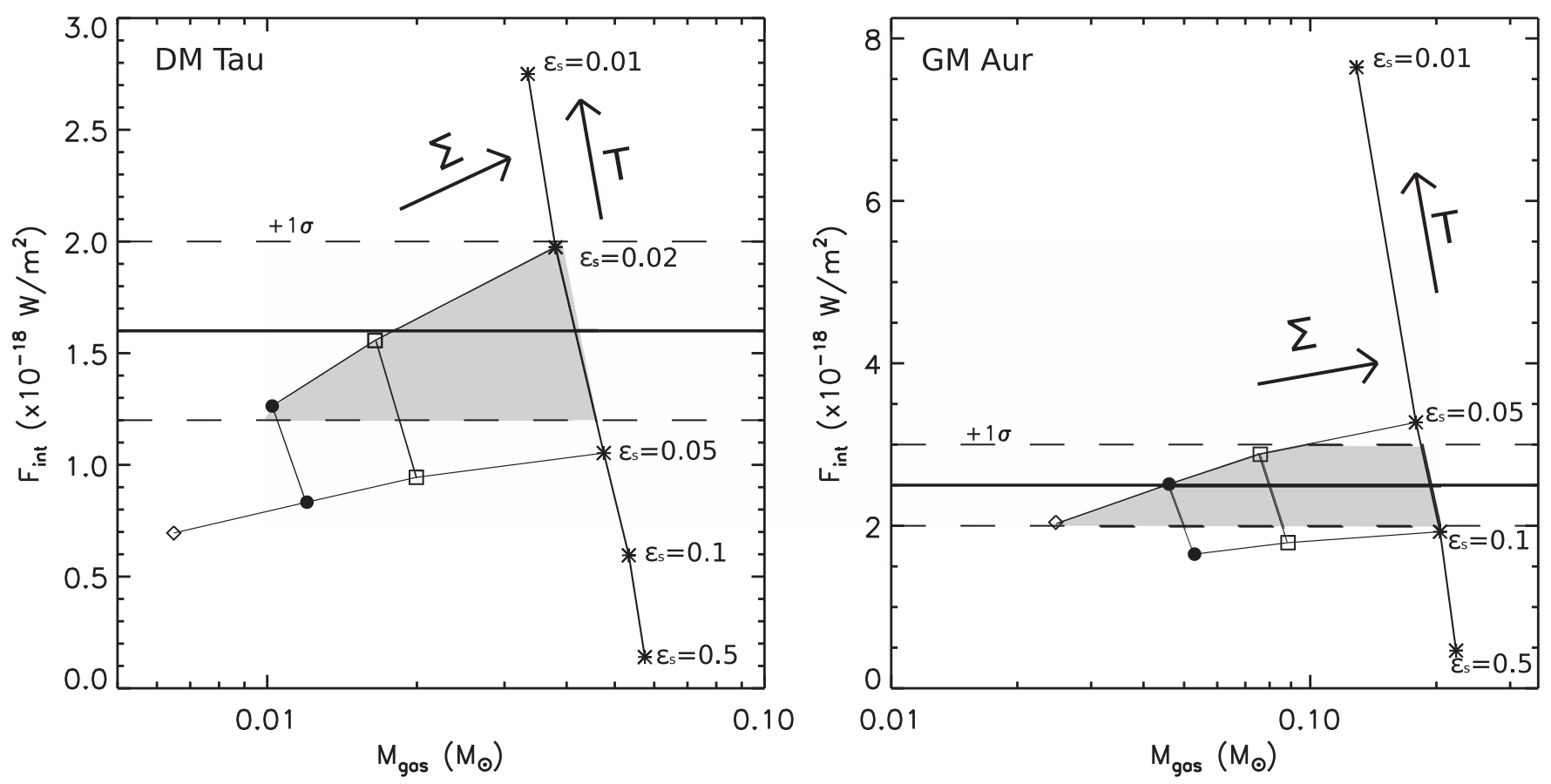

Figure 6. Integrated line flux as a function of total gas mass in a grid of different upper-layer temperature structures and disk surface densities for DM Tau (left) and GM Aur (right). The range of disk gas masses permitted by the SED fitting and the HD $J=1-0$ line flux is indicated by the gray fill. Arrows indicate the direction in the grid along which the temperature structure and surface density vary the most. Different symbols denote models with decreasing surface density and increasing midplane dust concentration: $\alpha=0.001$ and $\epsilon_{\text {big }}$ defined by vertical mass conservation (asterisks), $\alpha=0.002$ and $\epsilon_{\text {big }}=25$ (open squares), $\alpha=0.003$ and $\epsilon_{\text {big }}=50$ (filled circles), and $\alpha=0.005$ and $\epsilon_{\text {big }}=75$ (open diamonds). UV gas heating provides an additional 4-6\% increase in the line emission. The best-fitting mass ranges are $1.9 \times 10^{-2}$ to $4.3 \times 10^{-2} M_{\odot}$ for DM Tau and $4.5 \times 10^{-2}$ to $19.5 \times 10^{-2} M_{\odot}$ for GM Aur, although values of $1.0-4.7 \times 10^{-2}$ and $2.5-20.4 \times 10^{-2}$, respectively, are permitted within the $\pm 1 \sigma$ uncertainties.

seen by comparing the $\epsilon_{\text {small }}=0.02$ Case 2 family of models with those for $\epsilon_{\text {small }}=0.05$ for DM Tau in Figure 6. This is because a larger fraction of the HD is in the $J=1$ state for lower values of $\epsilon_{\text {small }}$, as explained above. The other disks in this sample have lower values of $\epsilon_{\text {small }}$, more consistent with the median value for Taurus $\left(\epsilon_{\text {small }}=0.01\right.$ Furlan et al. 2006). Thus the $J=1-0$ transition can be a good tracer of mass for a majority of disks, provided that the temperature structure is well constrained.

\subsection{Gas Masses}

Considering the full range of Case 1 and 2 models that fit both the SED and $1 \sigma$ uncertainties on the HD line flux, there is a range of masses for the disks with $\mathrm{HD}$ detections: $1.0-4.7 \times 10^{-2}$ for DM Tau and 2.5-20.4 $\times 10^{-2}$ for GM Aur, as given in Table 5. Although the mass for GM Aur is large, the disk is still marginally stable, with Toomre $\mathrm{Q} \sim 1.3$ at $300 \mathrm{au}$. The masses corresponding to the model grid are shown in Figure 6. The gray polygon indicates the parameter space that fits both the dust SED and the gas HD line. The maximum values for $\alpha$ and the increased dust/gas ratio were not physically motivated; to see how small a mass we could obtain, we increased these parameters until the models no longer fit the SED without considering whether such large values were realistic. Even with these extreme values, DM Tau's mass is greater than or equal to the minimum mass solar nebula. The mass of GM Aur is larger by at least a factor of two, even with the smallest surface density.

The masses we derive for these two disks may be underestimated because of our model assumption of total dust and gas depletion in their gaps. In contrast to this assumption, recent
ALMA observations have detected gas inside the dust gap for several disks in the transitional disk class with depletions of two to three orders of magnitude relative to the gap edge (van der Marel et al. 2016). If this is also the case for DM Tau and GM Aur, then in this work we have underestimated their disk gas masses.

Compared with the mass published by Bergin et al. (2013) for TW Hya, $0.06 M_{\odot}$, DM Tau has a mass that is $20 \%$ lower. However, when corrected for the difference in distance, DM Tau has a $64 \%$ brighter line detection. This discrepancy is likely due to a difference in the dust distribution in the upper layers, which has a large effect on the line flux, as demonstrated by Figure 6. If TW Hya is less settled than DM Tau, it would have a cooler midaltitude temperature structure and therefore a lower line flux for the same gas mass.

\subsection{Nondetections}

For the disks in which HD was not detected, we conducted a Case 1 model analysis in the same way as for DM Tau and GM Aur, testing the full $\epsilon_{\text {small }}$ and $\alpha$ parameter space. The HD line flux for the best fit to the SED was calculated and compared with the upper limit on the observed line flux listed in Table 2. The best-fitting SED models for two of these disks produce HD line fluxes that are formally just above the observed $3 \sigma$ upper limits to their integrated flux. Their peak central fluxes are still below the $3 \sigma$ level of the continuum: VZ Cha $\left(2.5 \times 10^{-18} \mathrm{~W}\right.$ $\left.\mathrm{m}^{-2}\right)$ and AA Tau $\left(2.5 \times 10^{-18} \mathrm{~W} \mathrm{~m}^{-2}\right)$. This puts a firm lower limit on $\epsilon_{\text {small }}$, as a lower value would produce stronger $\mathrm{HD}$ line emission. Another way to view the limit on $\epsilon_{\text {small }}$ is as an upper limit to the gas/dust ratio in the disk upper layers. For 


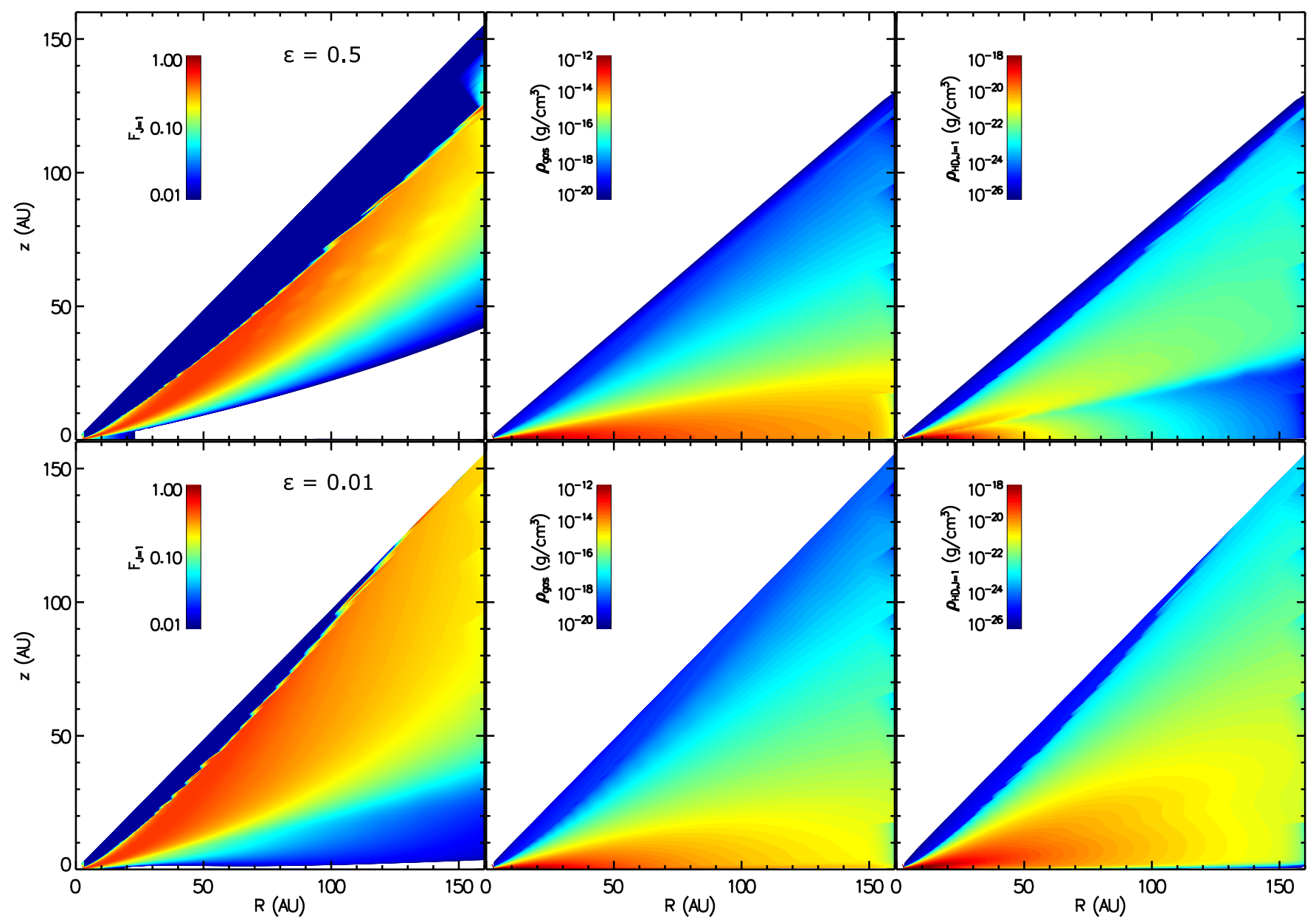

Figure 7. DM Tau disk structures. Upper row: fractional HD population in the $J=1$ level (left), gas density structure (center), and HD $J=1 \mathrm{density} \mathrm{structure} \mathrm{(right)}$ for $\epsilon_{\text {small }}=0.5$ (50\% less dust). Lower row: fractional HD population in the $J=1$ level (left), gas density structure (center), and HD $J=1$ density structure (right) for $\epsilon_{\text {small }}=0.01(99 \%$ less dust).

VZ Cha, the gas/dust mass ratio can be no larger than 153,800, while for AA Tau it must be lower than 15,380.

Since the maximum value of $\epsilon_{\text {small }}$ is better constrained for these two disks than for DM Tau and GM Aur (as they are thought to be full, nontransitional disks), and the value of the dust/gas ratio in the midplane of the Case I models is a lower limit for our parameter space, the total masses for these disks listed in Table 4 are true upper limits. The other two disks have integrated line fluxes well below their $3 \sigma$ upper limits from Table 2: FZ Tau $\left(1.8 \times 10^{-18} \mathrm{~W} \mathrm{~m}^{-2}\right)$ and LkCa 15 $\left(6.7 \times 10^{-19} \mathrm{~W} \mathrm{~m}^{-2}\right)$. For these disks, the derived total mass is an upper limit as well, simply due to the limits on $\epsilon_{\text {small }}$ provided by the SED fitting; however, the HD line flux itself imposes no additional constraints.

\section{DISCUSSION}

\subsection{Comparing CO and HD Gas Masses: Carbon Depletion?}

The range of gas masses found here results directly from the strong dependence of the HD line emission on both the gas temperature and density structures. Masses derived from $\mathrm{CO}$ exhibit similar dependencies, with the additional uncertainty of freeze-out and chemical sequestration of carbon into other, more complex species (Bruderer et al. 2012; Bruderer 2013; Favre et al. 2013; Bergin et al. 2014; Reboussin et al. 2015; Kama et al. 2016a, 2016b). Gas masses can be further underestimated if isotope-selective photodissociation is not properly taken into account (Miotello et al. 2014). Contrasting our gas masses with those derived from $\mathrm{CO}$ could independently confirm the chemical depletion of carbon in these disks.

For both DM Tau and GM Aur, gas masses of $1.4 \times 10^{-3}$ and $\leqslant 0.35 \times 10^{-3}$, respectively, were derived from $\mathrm{CO}$ measurements by Dutrey et al. (1996). This work did not account for either freeze-out, photodissociation, or chemical depletion, and the fact that their gas masses are at least an order of magnitude less than what we measure with $\mathrm{HD}$ is consistent with the expectation that the effects of freeze-out and photodissociation biases the COderived gas masses to lower values. A more recent work, Williams $\&$ Best (2014), calculates a disk gas mass from CO using a generic model grid that takes these two effects into account and compares this grid with a sample of disks that includes DM Tau only. Although Williams \& Best (2014) take into account optical depth effects by using $\mathrm{CO}$ isotopologs and parameterizing freeze-out and photodissociation, in the locations where $\mathrm{CO}$ should be present in the gas phase, they use a single value of $\mathrm{CO} / \mathrm{H}_{2}=1 \times 10^{-4}$ to determine the final gas mass. The mass that they derive from $\mathrm{CO}$ is consistent with our lower limit $\left(0.9 \times 10^{-2}\right.$ versus $1.0 \times 10^{-2}$ $M_{\odot}$ ) but five times lower than our upper limit of $4.7 \times 10^{-2} M_{\odot}$. This suggests that, even after photodissociation and freeze-out are accounted for, the CO in DM Tau may be chemically depleted by up to a factor of five. 


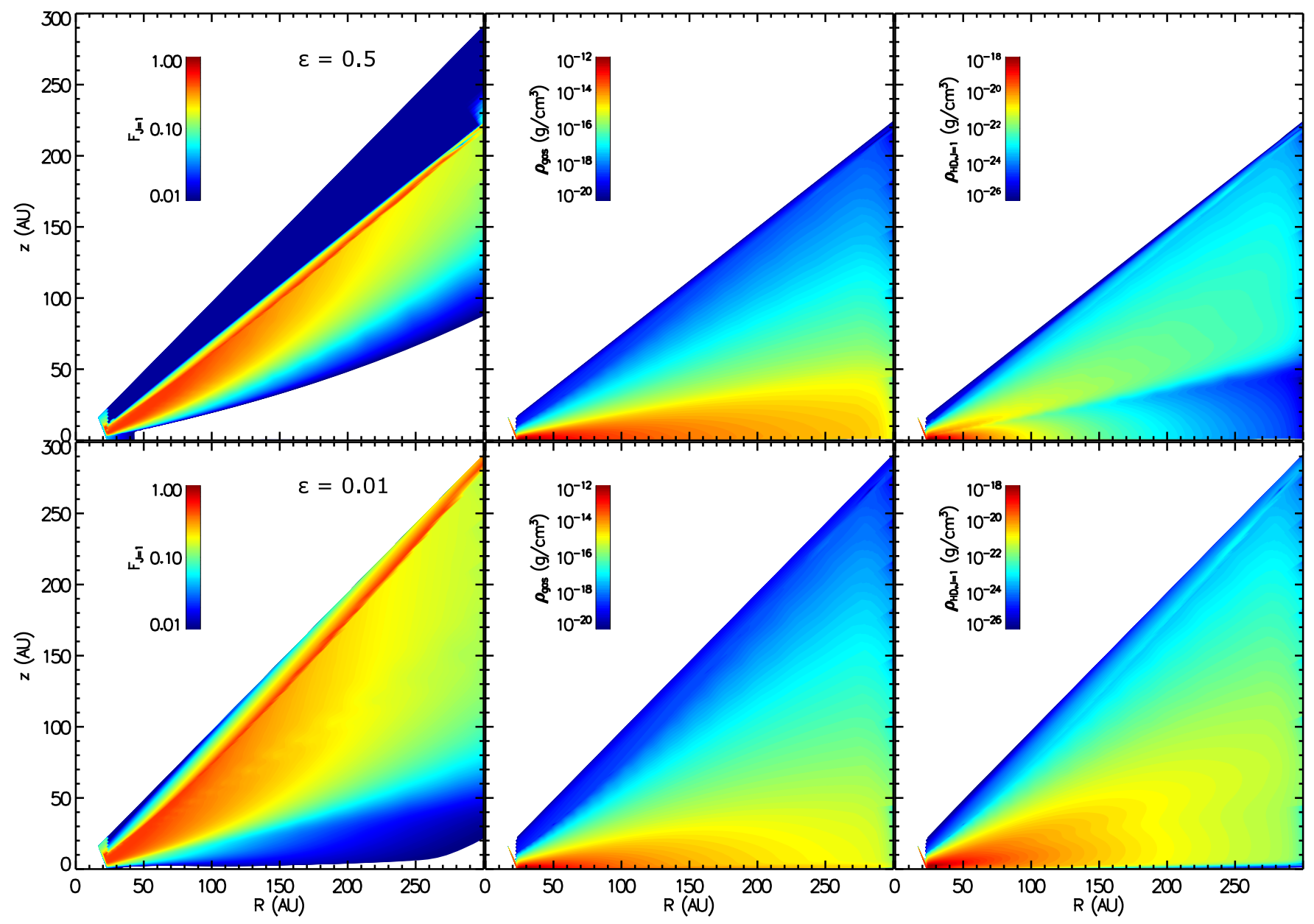

Figure 8. GM Aur disk structures. Upper row: fractional HD population in the $J=1$ level (left), gas density structure (center), and HD $J=1 \mathrm{density}$ structure (right) for $\epsilon_{\text {small }}=0.5$ (50\% less dust). Lower row: fractional HD population in the $J=1$ level (left), gas density structure (center), and HD $J=1$ density structure (right) for $\epsilon_{\text {small }}=0.01(99 \%$ less dust).

While we cannot entirely rule out their CO-derived gas mass, it is worth noting a few caveats. First, as discussed in Section 4.3, our lower limit to the mass requires a dust/gas enhancement at the midplane that is not physically motivated. Second, the gas masses derived from neither CO nor HD account completely for the presence of depleted gas and dust in the gaps or inner clearings of these transitional disks. Our work assumes a totally depleted gap, resulting in an underestimate of the disk mass, as described in Section 4.3. The CO gas masses were derived with the assumption of a full disk, without gaps or clearings, and therefore the gas mass is generally overestimated. Some secondary effects would also apply: the warmer midplane gas temperatures in the gap should prevent $\mathrm{CO}$ freeze-out there, while the additional dust depletion and greater UV penetration might allow more photodissociation. If those two effects cancel each other, then overall the modeled $\mathrm{CO}$ gas mass would be lower, while our mass should be higher.

While GM Aur was not modeled by Williams \& Best (2014), their gas mass for DM Tau was a factor of six greater than the mass found by Dutrey et al. (1996), which did not include freeze-out or photodissociation. Extrapolating the gas mass found for GM Aur by Dutrey et al. (1996) by the same factor results in a mass of $2 \times 10^{-3}$, which would yield a carbon depletion on the order of one to two orders of magnitude. This suggests that GM Aur could be more strongly depleted in carbon than DM Tau.

\subsection{Breaking the Degeneracies between Temperature Structure and Total Gas Mass}

In this work, we used a basic dust composition of silicates and graphite with a range of dust/gas ratios and showed how the disk temperature structure and gas mass could vary to produce different fits to the HD line. To constrain further the disk mass distribution and compare it against similar theoretical studies, it is necessary to combine these HD measurements with additional gas and dust data at sufficiently high spatial or spectral resolution to break the current degeneracies between the temperature structure and total gas mass. Since the gas and dust are still coupled at the disk height from which most of the HD $J=1$ level emits, accurate knowledge of the temperature structure requires understanding the distribution of dust in the upper layers and disk midplane.

The vertical temperature structure of a disk depends on the degree of dust settling and the detailed dust composition, both of which can be better constrained by continuum spectral data, such as from SPIRE observations, than they can be by continuum photometry as we have done here. An analysis of the detailed shape of the SED between 100 and $300 \mu \mathrm{m}$ will allow a more precise determination of $\epsilon_{\text {small }}$ and the dust properties (C. Espaillat et al. 2016, in preparation). Another way to probe specifically the vertical gas temperature structure in the region of the disk where HD emits is through line observations 
of CO. Simultaneous modeling of the CO ladder in the far infrared has constrained the temperature structure in the upper layers of several bright disks (Fedele et al. 2013, 2016). At longer wavelengths, Rosenfeld et al. (2013) find evidence for the vertical location of the $\mathrm{CO}$ freeze-out zone (typically $\left.T_{\text {dust }} \sim 20 \mathrm{~K}\right)$ and the location of the surface where ${ }^{12} \mathrm{CO}$ becomes optically thick. These types of measurements, in conjunction with less optically thick $\mathrm{CO}$ isotopologues, could be used to pin down the temperature structure at different depths in the disk (Cleeves et al. 2015; Kama et al. 2016a, 2016b; Miotello et al. 2016). Additionally, observations of the $J=6-5$ transition of ${ }^{13} \mathrm{CO}$, which has an upper state energy similar to the HD $J=1-0$ transition, can resolve the temperature structure over a region similar to the HD transition (Schwarz et al. 2016). Once the temperature structure is determined, a more precise gas mass can be determined from Figure 6 .

Another way to constrain the mass in Figure 6 is to combine the HD measurements with constraints on the midplane temperature structure from resolved observations of the $\mathrm{CO}$ snow line. Varying the dust/gas ratio changes the midplane dust temperature. For example, GM Aur models in which the vertical dust mass has been conserved have the $\mathrm{CO}$ snow line $(T=20 \mathrm{~K})$ at $51-53 \mathrm{au}$. These are the models with the largest disk gas mass. At the other end of the mass range, with models that include a dust/gas ratio enhanced by some additional effect (e.g., radial drift), the CO snow line lies at 44 au. ALMA has already provided resolved observations of the $\mathrm{CO}$ snow line for some disks (Qi et al. 2013); similar observations would put strong constraints on the midplane dust/gas ratio and hence the total gas mass.

\section{CONCLUSIONS}

In this work we have detected the HD $J=1-0$ transition in two T Tauri disks, DM Tau and GM Aur, out of a sample of six. Using a combination of SED and line emission models, we determine the ranges of mass permitted to these disks, given the uncertainties in the temperature structure and dust/gas ratio from the observational uncertainties.

1. DM Tau and GM Aur are found to have masses of $1.0-4.7 \times 10^{-2}$ and $2.5-20.4 \times 10^{-2}$, respectively.

2. For the disks in which HD is not detected, the nondetections provide strict limits on the gas/dust ratio in the disk upper layers of $<153,800$ and $<15,380$ for VZ Cha and AA Tau, respectively, while limits could be established for LkCa15 and FZ Tau only on the gas/dust ratio from SED fitting.

3. A comparison of our HD gas masses and those derived from CO suggests that GM Aur shows gas-phase carbon depletion of up to two orders of magnitude, while DM may show depletion of up to a factor of five.

Going forward, a combination of physically motivated models with disk chemistry that fit $\mathrm{HD}, \mathrm{CO}$, and the dust SED will be able to disentangle the disk temperature and density structures while determining the degree of carbon depletion. Observations of the HD $J=1-0$ line are a particularly useful way to constrain the disk mass; future facilities like SPICA will hopefully make this transition available for more sources.

This work is based on observations made with Herschel, a European Space Agency Cornerstone Mission with significant participation by NASA. Support for this work was provided by NASA through an award issued by JPL/Caltech. We thank Davide Fedele for useful discussions regarding the data reduction and line flux determinations. We would also like to thank the anonymous referee for constructive suggestions.

\section{APPENDIX \\ FRACTIONAL POPULATIONS OF HD IN THE $J=1$ STATE}

In Figures 7 and 8 we display the fractional population and mass density in the HD $\mathrm{J}=1$ state compared with the mass density of the total disk for the most extreme cases of dust settling tested for DM Tau and GM Aur. With stronger dust settling, the HD $J=1$ line probes the full gas reservoir of the disk.

\section{REFERENCES}

Adams, F. C., Emerson, J. P., \& Fuller, G. A. 1990, ApJ, 357, 606

Andrews, S. M., \& Williams, J. P. 2005, ApJ, 631, 1134

Andrews, S. M., \& Williams, J. P. 2007a, ApJ, 671, 1800

Andrews, S. M., \& Williams, J. P. 2007b, ApJ, 659, 705

Andrews, S. M., \& Williams, J. P. 2008, Ap\&SS, 313, 119

Andrews, S. M., Wilner, D. J., Espaillat, C., et al. 2011, ApJ, 732, 42

Andrews, S. M., Wilner, D. J., Hughes, A. M., et al. 2012, ApJ, 744, 162

Armitage, P. J. 2011, ARA\&A, 49, 195

Beckwith, S. V. W., \& Sargent, A. I. 1991, ApJ, 381, 250

Beckwith, S. V. W., Sargent, A. I., Chini, R. S., \& Guesten, R. 1990, AJ, 99, 924

Bergin, E. A., Cleeves, L. I., Crockett, N., \& Blake, G. A. 2014, FaDi, 168, 61

Bergin, E. A., Cleeves, L. I., Gorti, U., et al. 2013, Natur, 493, 644

Bethell, T., \& Bergin, E. 2009, Sci, 326, 1675

Bruderer, S. 2013, A\&A, 559, A46

Bruderer, S., van Dishoeck, E. F., Doty, S. D., \& Herczeg, G. J. 2012, A\&A, 541, A91

Calvet, N., D’Alessio, P., Watson, D. M., et al. 2005, ApJL, 630, L185

Carpenter, J. M., Hillenbrand, L. A., Skrutskie, M. F., \& Meyer, M. R. 2002, AJ, 124, 1001

Chiang, E. I., Joung, M. K., Creech-Eakman, M. J., et al. 2001, ApJ, 547, 1077

Chihara, H., Koike, C., Tsuchiyama, A., Tachibana, S., \& Sakamoto, D. 2002, A\&A, 391, 267

Cleeves, L. I., Bergin, E. A., Qi, C., Adams, F. C., \& Öberg, K. I. 2015, ApJ, 799, 204

Cox, A. W., Grady, C. A., Hammel, H. B., et al. 2013, ApJ, 762, 40

Cutri, R. M., Skrutskie, M. F., van Dyk, S., et al. 2003, yCat, 2246, 0

Cutri, R. M., Wright, E. L., Conrow, T., et al. 2012, yCat, 2311, 0

D’Alessio, P., Calvet, N., Hartmann, L., Franco-Hernández, R., \& Servín, H. 2006, ApJ, 638, 314

D’Alessio, P., Calvet, N., Hartmann, L., Muzerolle, J., \& Sitko, M. 2004, in IAU Symp. 221, Star Formation at High Angular Resolution, ed. M. G. Burton, R. Jayawardhana, \& T. L. Bourke (Cambridge: Cambridge Univ. Press), 403

Dorschner, J., Begemann, B., Henning, T., Jaeger, C., \& Mutschke, H. 1995, A\&A, 300, 503

Draine, B. T., \& Lee, H. M. 1984, ApJ, 285, 89

Dutrey, A., Guilloteau, S., Duvert, G., et al. 1996, A\&A, 309, 493

Dutrey, A., Guilloteau, S., \& Guelin, M. 1997, A\&A, 317, L55

Dutrey, A., Guilloteau, S., Prato, L., et al. 1998, A\&A, 338, L63

Duvert, G., Guilloteau, S., Ménard, F., Simon, M., \& Dutrey, A. 2000, A\&A, 355,165

Espaillat, C., D’Alessio, P., Hernández, J., et al. 2010, ApJ, 717, 441

Espaillat, C., Furlan, E., D’Alessio, P., et al. 2011, ApJ, 728, 49

Favre, C., Cleeves, L. I., Bergin, E. A., Qi, C., \& Blake, G. A. 2013, ApJL, 776, L38

Fedele, D., Bruderer, S., van Dishoeck, E. F., et al. 2013, ApJL, 776, L3

Fedele, D., van Dishoeck, E. F., Kama, M., Bruderer, S., \& Hogerheijde, M. R. 2016, A\&A, 591, A95

Furlan, E., Hartmann, L., Calvet, N., et al. 2006, ApJS, 165, 568

Gräfe, C., Wolf, S., Roccatagliata, V., Sauter, J., \& Ertel, S. 2011, A\&A, 533, A89

Green, J. D., Yang, Y.-L., Evans, N. J., II, et al. 2016, AJ, 151, 75

Guilloteau, S., Dutrey, A., Piétu, V., \& Boehler, Y. 2011, A\&A, 529, A105 
Hayashi, C. 1981, PThPS, 70, 35

Helou, G., \& Walker, D. W. (ed.) 1988, Infrared Astronomical Satellite (IRAS) Catalogs and Atlases: The Small Scale Structure Catalog, Vol. 7

Herbig, G. H., \& Bell, K. R. 1988, Third Catalog of Emission-Line Stars of the Orion Population

Howard, C. D., Sandell, G., Vacca, W. D., et al. 2013, ApJ, 776, 21

Ingleby, L., Espaillat, C., Calvet, N., et al. 2015, ApJ, 805, 149

Isella, A., Carpenter, J. M., \& Sargent, A. I. 2009, ApJ, 701, 260

Ishihara, D., Onaka, T., Kataza, H., et al. 2010, A\&A, 514, A1

Jaeger, C., Mutschke, H., Begemann, B., Dorschner, J., \& Henning, T. 1994, A\&A, 292, 641

Kama, M., Bruderer, S., Carney, M., et al. 2016a, A\&A, 588, A108

Kama, M., Bruderer, S., van Dishoeck, E. F., et al. 2016b, A\&A, 592, A83

Kenyon, S. J., \& Hartmann, L. 1995, ApJS, 101, 117

Kitamura, Y., Momose, M., Yokogawa, S., et al. 2002, ApJ, 581, 357

Linsky, J. L. 1998, SSRv, 84, 285

Luhman, K. L., Allen, L. E., Allen, P. R., et al. 2008, ApJ, 675, 1375

Manara, C. F., Fedele, D., Herczeg, G. J., \& Teixeira, P. S. 2016, A\&A, 585, A136

Mathis, J. S., Rumpl, W., \& Nordsieck, K. H. 1977, ApJ, 217, 425

McClure, M. K., D'Alessio, P., Calvet, N., et al. 2013, ApJ, 775, 114

McClure, M. K., Espaillat, C., Calvet, N., et al. 2015, ApJ, 799, 162

Miotello, A., Bruderer, S., \& van Dishoeck, E. F. 2014, A\&A, 572, A96

Miotello, A., van Dishoeck, E. F., Kama, M., \& Bruderer, S. 2016, A\&A, 594, A85

Mordasini, C., Alibert, Y., Benz, W., Klahr, H., \& Henning, T. 2012, A\&A, 541, A97

Moshir, M., Kopan, G., Conrow, T., et al. 1990, IRAS Faint Source Catalogue, version 2.0

Osterloh, M., \& Beckwith, S. V. W. 1995, ApJ, 439, 288

Panić, O., Hogerheijde, M. R., Wilner, D., \& Qi, C. 2009, A\&A, 501, 269
Pilbratt, G. L., Riedinger, J. R., Passvogel, T., et al. 2010, A\&A, 518, L1

Poglitsch, A., Waelkens, C., Geis, N., et al. 2010, A\&A, 518, L2

Pontoppidan, K. M., Meijerink, R., Dullemond, C. P., \& Blake, G. A. 2009, ApJ, 704, 1482

Qi, C., Öberg, K. I., Wilner, D. J., et al. 2013, Sci, 341, 630

Reboussin, L., Wakelam, V., Guilloteau, S., Hersant, F., \& Dutrey, A. 2015, A\&A, 579, A82

Ricci, L., Testi, L., Natta, A., et al. 2010, A\&A, 512, A15

Rodmann, J., Henning, T., Chandler, C. J., Mundy, L. G., \& Wilner, D. J. 2006, A\&A, 446, 211

Rosenfeld, K. A., Andrews, S. M., Hughes, A. M., Wilner, D. J., \& Qi, C. 2013, ApJ, 774, 16

Sargent, B. A., Forrest, W. J., Tayrien, C., et al. 2009, ApJS, 182, 477

Schwarz, K. R., Bergin, E. A., Cleeves, L. I., et al. 2016, ApJ, 823, 91

Shakura, N. I., \& Sunyaev, R. A. 1973, A\&A, 24, 337

Sogawa, H., Koike, C., Chihara, H., et al. 2006, A\&A, 451, 357

Teague, R., Semenov, D., Guilloteau, S., et al. 2015, A\&A, 574, A137

Testi, L., Birnstiel, T., Ricci, L., et al. 2014, in Protostars and Planets VI, ed. H. Beuther, R. S. Klessen, C. P. Dullemond, \& T. Henning (Tuscon, AZ: Univ. Arizona Press), 339

van der Marel, N., van Dishoeck, E. F., Bruderer, S., et al. 2016, A\&A, 585, A58

van Zadelhoff, G.-J., van Dishoeck, E. F., Thi, W.-F., \& Blake, G. A. 2001, A\&A, 377, 566

Visser, R., van Dishoeck, E. F., \& Black, J. H. 2009, A\&A, 503, 323

Weintraub, D. A., Sandell, G., \& Duncan, W. D. 1989, ApJL, 340, L69

Williams, J. P., \& Best, W. M. J. 2014, ApJ, 788, 59

Winston, E., Cox, N. L. J., Prusti, T., et al. 2012, A\&A, 545, A145

Yang, H., Herczeg, G. J., Linsky, J. L., et al. 2012, ApJ, 744, 121

Zhu, Z., Nelson, R. P., Dong, R., Espaillat, C., \& Hartmann, L. 2012, ApJ, 755,6 